Article

\title{
A Predicted Mannoprotein Cmp1 Regulates Fungal Virulence in Cryptococcus neoformans
}

\author{
Lian-Tao Han ${ }^{1,2}$, Lei $\mathrm{Wu}^{1}$ and Tong-Bao Liu ${ }^{1,2, * \mathbb{D}}$ \\ 1 State Key Laboratory of Silkworm Genome Biology, Southwest University, Chongqing 400715, China; \\ hlt892996713@email.swu.edu.cn (L.-T.H.); 18215522009@163.com (L.W.) \\ 2 Chongqing Key Laboratory of Microsporidia Infection and Control, Southwest University, \\ Chongqing 400715, China \\ * Correspondence: tongbaoliu@swu.edu.cn; Tel.: +86-23-6825-1088
}

Received: 27 September 2020; Accepted: 23 October 2020; Published: 24 October 2020

\begin{abstract}
The capsule of the fungal pathogen Cryptococcus neoformans consists of glucuronoxylomannan (GXM), glucuronoxylomannogalactan (GXMGal), and mannoproteins (MPs). MPs are a kind of glycoproteins with low content but high immunogenicity, which can stimulate the immune protection of the host. However, there is not much information about the role of mannoproteins in virulence of the human fungal pathogen C. neoformans. In this study, we reported the identification and functional analysis of a predicted mannoprotein $\mathrm{Cmp1}$ that regulates fungal virulence in C. neoformans. Gene expression pattern analysis indicates that the CMP1 gene was ubiquitously expressed at all stages of cryptococcal development. Subcellular localization analysis indicated that $\mathrm{Cmp} 1$ was localized in the cytoplasm of cryptococcal cells. Disruption or overexpression of CMP1 results in impairing capsule formation in Cryptococcus, but it does not affect the melanin production and sensitivity under various stress conditions, nor does it affect the sexual reproduction process of Cryptococcus. Survival assay showed that the pathogenicity of the $\operatorname{cmp} 1 \Delta$ mutant or the $C M P 1$ overexpression strain was significantly attenuated in a murine inhalation model of cryptococcosis. In conclusion, our findings implied that the mannoprotein Cmp1 is required for the virulence of $C$. neoformans.
\end{abstract}

Keywords: Cryptococcus neoformans; mannoprotein; Cmp1; virulence; capsule

\section{Introduction}

Cryptococcus neoformans is an encapsulated yeast-like pathogen that can cross the blood-brain barrier to cause fungal meningitis predominantly in immunocompromised patients, leading to hundreds of thousands of deaths each year [1-3]. As a human pathogenic fungus, C. neoformans possess several virulence factors such as capsule and melanin, and growth at $37^{\circ} \mathrm{C}$, which contribute to the survival of $C$. neoformans in the host [4-6]. Among the virulence factors, polysaccharide capsules are one of the most crucial factors to cause disease $[7,8]$. The capsule is comprised of glucuronoxylomannan (GXM, 90\%), glucuronoxylomannogalactan (GXMGal, 9\%), and small proportions of mannoproteins (MPs, $1 \%$ ) $[7,9]$.

There are many kinds of MPs. Although the molecular weight of the MPs is different, they all have the conserved motifs, including a signal peptide, a serine/threonine (Ser/Thr)-rich region, a glycosylphosphatidylinositol (GPI) anchor attach site, and a functional domain [9]. These MPs contain abundant O-glycosylation sites in Ser/Thr rich regions [10-12]. Immunoelectron microscopy analysis showed that most MPs were located near the inner cell wall [13]. Although MPs' content in the polysaccharide capsule is small, it plays an important physiological role in fungi. Teixeira et al. demonstrated that the mannoprotein MP84 was involved in the C. neoformans-epithelial lung cells adhesion through a Cryptococcus-epithelial lung cell interaction assay [14]. The frequent 
occurrence of cryptococcosis in T-cell deficient patients underscores the importance of the host cell response, and antigens that can recognize and cause cell responses have attracted much attention [15-17]. C. neoformans MPs are highly immunogenic and can stimulate T-cell responses [12,18]. The mannoproteins MP88 and MP98 can stimulate T-cell responses, and the MP84 and MP115 proteins can react strongly with sera from AIDS patients infected by $C$. neoformans $[9,10,14,19]$, indicating that the MPs may be the main antigen of $C$. neoformans to stimulate the T-cell response. The immune mechanism of MPs may lie in its ability to target and bind the mannose receptors on the surface of antigen-presenting cells (APC), and it is speculated that this mechanism can induce not only a cellular immune response but also induce humoral response [20], which plays an important role in indicating the development of the cryptococcal vaccine.

Other mannoproteins also play an important role in virulence and/or cell wall integrity of fungal pathogens. Cadieux et al. showed that the $C$. neoformans mannoprotein Cig1 helps get iron from heme and further proved that the Cig1 and the ferroxidase Cfo1 jointly regulate the virulence of C. neoformans [21]. Reuwsaat et al. found that the predicted mannoprotein Krp1 is required for the cell wall structural integrity but is not necessary for fungal virulence in vivo in C. gattii [22]. Viudes et al. found that Candida albicans surface mannoprotein MP58 could elicit a strong antibody response during infection. The C-terminal antibody binding region of the MP58 protein is the antigenic epitopes of C. albicans, and monoclonal antibodies targeting this epitope can play a protective role in the serum treatment experiments in a mouse model of candidiasis [23]. In Candida glabrata, the mannoprotein Tir3 is necessary for sterol uptake [24]. In Talaromyces (Penicillium) marneffei, the mannoprotein Mp1p was proved to be an essential virulence factor, mediating virulence by enhancing T. marneffei's survival inside macrophages $[25,26]$.

The above results showed that although mannoprotein is not the main structural substance of fungal cells, it affects the growth, development, and virulence of fungal pathogens, and some mannoproteins can also act as specific antigens to stimulate the cellular immune response of the host and perform immune protection on the host.

In our previous work on identifying downstream targets of Fbp1, an F-box protein, we found a highly enriched protein Cmp1 (CNAG_06000) in fbp1 $\Delta$ mutant background. The protein sequence analysis indicated that this protein has a high similarity with the mannoprotein MP88 of C. neoformans and T. asahii, and might be a mannoprotein. Since mannoproteins can develop protective immunity, we hypothesized that $\mathrm{Cmp} 1$ is a virulence factor that regulates the virulence of $C$. neoformans. In the present study, we knocked out and overexpressed the CMP1gene and examined its virulence role in a murine model to test our hypothesis. We demonstrated that the mannoprotein $\mathrm{Cmp} 1$ affects capsule production and regulates fungal virulence in C. neoformans.

\section{Results}

\subsection{Identification of the Mannoprotein Cmp1 in C. neoformans}

In our previous study, we used an iTRAQ-based proteomics approach to identify the downstream targets of Fbp1, a key protein of the E3 ubiquitin ligase. One of the candidate proteins, CNAG_06000, might be a substrate of Fbp1 because of the putative PEST (proline (P), glutamate (E), serine (S), and threonine $(\mathrm{T})$ ) domains in its protein sequences (Table 1, Figure 1). To investigate the function of cryptococcal CNAG_06000, we blasted the gene against the FungiDB database [27] and found that the CNAG_06000 gene is 1968 bp long and consists of 8 exons encoding a protein of 489 amino acids (Figure 1A, B). Analysis of the CNAG_06000 amino acid sequence showed that it contains one Ser-rich domain (Ser_RICH), one transmembrane domain (TR), five N-linked glycosylation sites (NGS), and one glycosyl-phosphatidylinositol (GPI)-anchor site ( $\omega$; Figure 1B). Smart blast analysis revealed that the CNAG_06000 protein shows $68 \%$ and 70\% sequence similarities to the mannoprotein MP88 in Trichosporon asahii and C. neoformans, respectively (Figure 1C); we named the protein Cmp1 (Cryptococcus mannoprotein 1). The further protein sequences analysis indicates that the Cmp1 lacks 
signal peptide compared with the MP88s in C. neoformans and T. asahii. Cryptococcus MP88, as a specific antigen, stimulates the host's cellular immune response and plays an immunoprotective role on the host [19]. Due to the importance of mannoproteins in the C. neoformans-host interaction, we decided to explore the function of Cmp1 in fungal development and virulence of $C$. neoformans.

Table 1. High-abundance proteins identified in the $f b p 1 \Delta$ mutants.

\begin{tabular}{|c|c|c|c|}
\hline Accession & Description & Average $f b p 1 \Delta / \mathrm{H} 99$ & PEST Domain \\
\hline CNAG_00626 & Uncharacterized protein & 3.02656981 & 1 \\
\hline CNAG_06000 & Glycoprotein & 1.87701854 & 1 \\
\hline CNAG_06195 & Uncharacterized protein & 1.59340506 & 0 \\
\hline CNAG_05395 & Rab guanyl-nucleotide exchange factor & 1.5884178 & 3 \\
\hline CNAG_00700 & Purine nucleotide biosynthesis-related protein & 1.54435399 & 0 \\
\hline CNAG_02455 & Choline transporter & 1.46895293 & 0 \\
\hline CNAG_06871 & Uncharacterized protein & 1.45686983 & 1 \\
\hline CNAG_02138 & DNA replication ATP-dependent helicase Dna2 & 1.44213261 & 2 \\
\hline CNAG_01536 & Nonmuscle myosin heavy chain $\mathrm{b}$ & 1.3779496 & 1 \\
\hline CNAG_04056 & Rhomboid-like protein & 1.37338159 & 0 \\
\hline CNAG_04669 & Mitochondrial matrix protein import protein & 1.36855445 & 0 \\
\hline CNAG_03099 & Chitin synthase 1 & 1.36597408 & 1 \\
\hline CNAG_01193 & Uncharacterized protein & 1.36434725 & 1 \\
\hline CNAG_05817 & GDP-mannose transporter 1 & 1.35714493 & 0 \\
\hline CNAG_05967 & Uncharacterized protein & 1.35536384 & 1 \\
\hline CNAG_04327 & SCP160 protein & 1.35312155 & 1 \\
\hline CNAG_00634 & Uncharacterized protein & 1.3519146 & 1 \\
\hline CNAG_05173 & DNA-3-methyladenine glycosylase II & 1.34218534 & 0 \\
\hline CNAG_03281 & Glycine-rich RNA binding protein, variant 2 & 1.33612571 & 0 \\
\hline
\end{tabular}

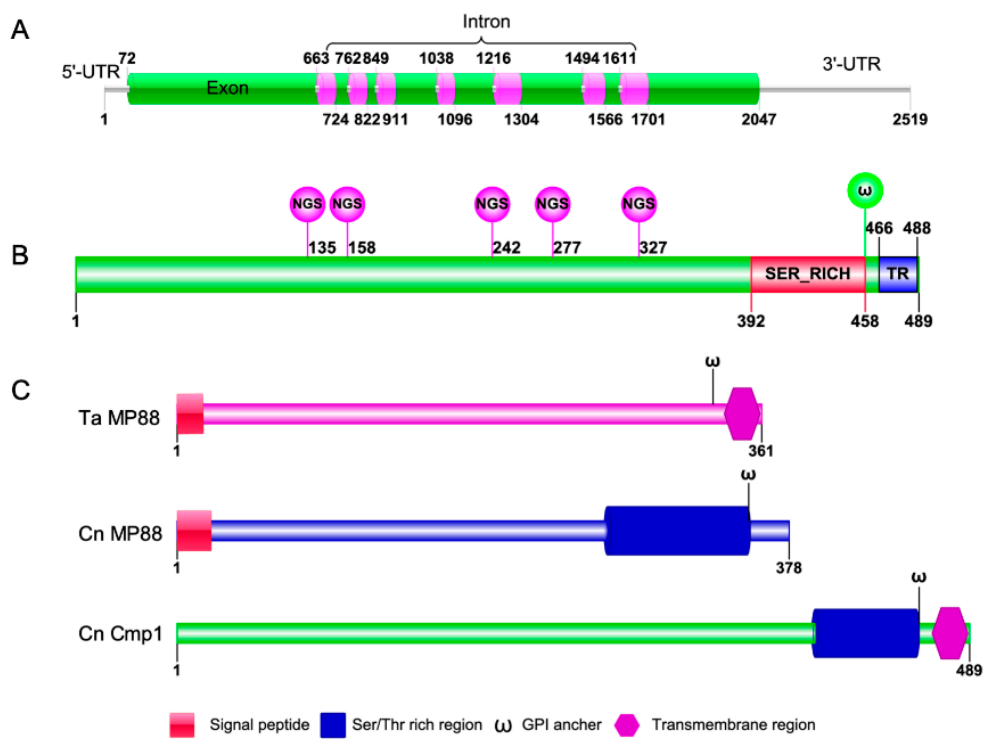

Figure 1. Sequence analysis of the Cryptococcus mannoprotein Cmp1. (A) The sequence structure of the Cryptococcus mannoprotein encoding gene CMP1. (B) Functional domains or sites of the Cryptococcus mannoprotein Cmp1. NGS: Glycosylation site; SER_RICH: ser/thr rich region; $\omega$ : GPI anchor; TR: Transmembrane region. (C) Comparison of protein structures between C. neoformans $\mathrm{Cmp} 1$ (Cn Cmp1), C. neoformans MP88 (Cn MP88), and T. asahil MP88 (Ta MP88).

\subsection{CMP1 Expression Pattern Analyses}

To analyze the Cryptococcus CMP1 gene expression pattern, we cloned a $1.7 \mathrm{~Kb}$ native promoter of the CMP1 gene first and fused it with mCherry $\left(P_{C M P 1}-m C h e r r y\right)$ to explore the temporal characteristics of the CMP1 gene expression. The mCherry signals were detected in yeast cells, mating hyphae, basidium, and spores of $\mathrm{P}_{\mathrm{CMP} 1}$-mCherry strains (TBL209 and TBL175, see Table 2 for detailed information), 
indicating that the CMP1 gene was expressed in all stages of Cryptococcus development (Figure 2A). Meanwhile, the expression of CMP1 during mating was also analyzed by qRT-PCR. The mating mixtures between H99 (MAT $\alpha$ ) and KN99 (MATa), the two wild-type strains, were harvested from V8 plates after designated incubation times. Compared with the 0 -h time point's gene expression level, our qRT-PCR data revealed that the $C M P 1^{\prime}$ s expression was first upregulated and then downregulated during the process of mating, suggesting its possible role in the sexual reproduction of $C$. neoformans (Figure 2B). Since Cmp1 might be a mannoprotein, we then tested the expression of the CMP1 gene under the capsule-inducing conditions, and the results showed that when Cryptococcus was cultured in YNB (yeast nitrogen base without amino acids) or DME (Dulbecco's modified Eagle) medium, the expression of the CMP1 gene was highly induced compared with that of CMP1 in YPD (yeast extract peptone dextrose). However, when Cryptococcus was cultured in the MM (minimal medium) medium, the expression levels of the CMP1 gene showed no difference from that of Cryptococcus cultured in the YPD medium (Figure 2C).

Table 2. Strains and plasmids used in this study.

\begin{tabular}{|c|c|c|}
\hline Strains/Plasmids & Genotypes/Properties & Sources/References \\
\hline \multicolumn{3}{|c|}{ C. neoformans } \\
\hline H99 & $M A T \alpha$ & Perfect et al., 1993 [28] \\
\hline KN99a & MATa & Nielsen et al., 2003 [29] \\
\hline TBL106 & $M A T \alpha \operatorname{cmp} 1 \triangle \because: N E O$ & In this study \\
\hline TBL137 & MATа стp $1 \triangle:: N E O$ & In this study \\
\hline TBL175 & MATa $P_{C M P 1}-m$ Cherry::NAT & In this study \\
\hline TBL177 & $M A T \alpha$ cmp $1 \triangle:: N E O C M P 1:: N A T$ & In this study \\
\hline TBL178 & MATа стр1 $1:: N E O$ CMP1::NAT & In this study \\
\hline TBL186 & $M A T \alpha c m p 1 \Delta:: N E O P_{H 3}-G F P-C M P 1:: N A T$ & In this study \\
\hline TBL187 & MATа cmp1 $1:: N E O P_{H 3-} G F P-C M P 1:: N A T$ & In this study \\
\hline \multicolumn{2}{|r|}{ Plasmids } & In this study \\
\hline pCN19 & $\mathrm{Amp}^{\mathrm{r}}$ Vector carrying GFP under the control of histone $\mathrm{H} 3$ promoter & Price et al., 2008 [30] \\
\hline pTBL1 & Amp ${ }^{r}$ Vector carrying NAT marker & Fan et al., 2019 [31] \\
\hline pTBL3 & Amp ${ }^{r}$ Vector carrying $m$ Cherry-GPD1 terminator & Fan et al., 2019 [31] \\
\hline pTBL82 & $A m p^{r}$ Vector carrying $P_{C M P 1}-m C h e r r y-N A T$ for temporal expression assay & In this study \\
\hline pTBL92 & Amp ${ }^{\mathrm{r}}$ Vector carrying $P_{H 3}-G F P-C M P 1$ gene fusion for Cmp1 localization & In this study \\
\hline pTBL94 & Amp ${ }^{\mathrm{r}}$ Vector carrying $P_{C M P 1}-C M P 1-N A T$ for $C M P 1$ complementation & In this study \\
\hline
\end{tabular}

\subsection{Cmp1 Localization in C. neoformans}

To detect the subcellular localization of cryptococcal Cmp1, we constructed the GFP-CMP1 fusion expression vector (pTBL92) and transformed it into the two cmp1 1 mutant strains (TBL106 and TBL137). Different from GFP-Nop1's nuclear localization (TBL84) [32] (Figure 3A), the fusion GFP-Cmp1 protein was distributed unevenly in the cytoplasm of Cryptococcus yeast cells and mating hyphae (Figure 3A). Next, we also examined the cellular localization of GFP-Cmp1 fusion protein under various stress conditions including high-temperature, hyperosmosis, oxidative, or nitrosative stresses. Our data suggested that the subcellular localization of GFP-Cmp1 did not change under the above stress conditions (Figure 3B). Meanwhile, we also examined the cellular localization of GFP-Cmp1 under capsule induction conditions. Interestingly, we found that the GFP-Cmp1 fusion protein localized in the vacuole of cryptococcal yeast cells (Figure 3C).

Since MM medium is a nutrient starvation medium used for capsule induction, it may affect the cellular localization of GFP-Cmp1. We then examined the cellular localization of the GFP-Cmp1 fusion protein in media such as YNB and DMEM. Our results showed that the GFP-Cmp1 fusion protein was localized in the cytoplasm of cryptococcal cells when the fluorescent strain was cultured in the YPD, YNB, or DME medium, while the GFP-Cmp1 fusion protein was localized in the vacuole when the fluorescent strain was cultured in the MM medium (Figure 3D). Our results indicate that the Cmp1 protein does have different subcellular localization under different culture conditions. 


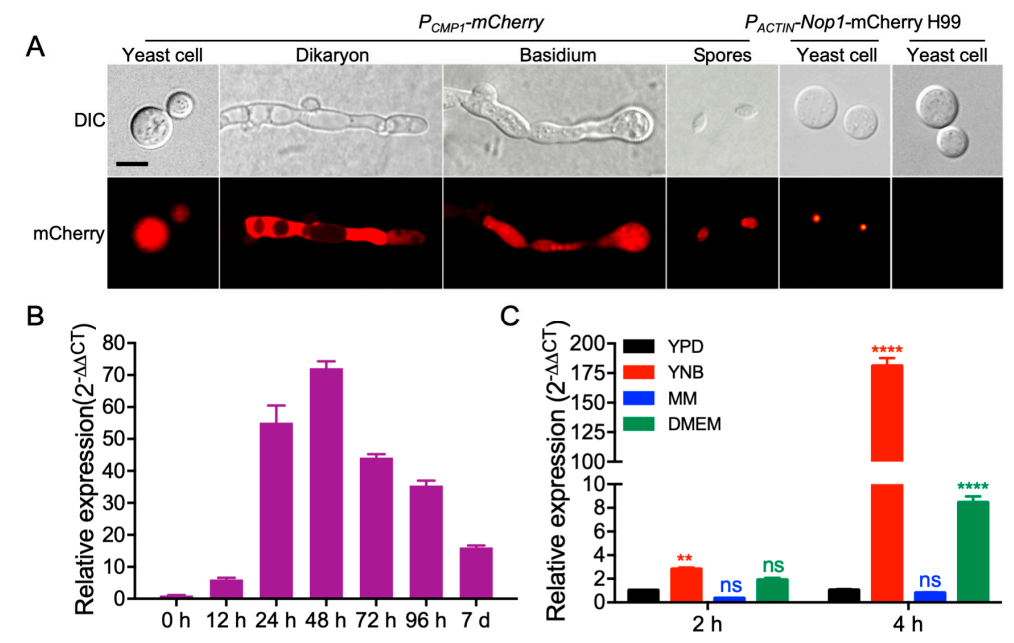

Figure 2. The expression pattern of the cryptococcal CMP1 gene. (A) The expression of $P_{C M P 1}-m C h e r r y$ during the mating was visualized by confocal microscopy (Olympus, FV1200, Tokyo, Japan). Representative images of yeast cells, mating hyphae, basidia, and basidiospores of both bright-field and fluorescence are shown. Scale bar, $5 \mu \mathrm{m}$. (B) Quantitative real-time PCR (qRT-PCR) was used to analyze the expression of CMP1 during mating. Mating mixtures of the wild-type strains were harvested from the V8 plates after incubation for indicated times and used for RNA extraction, and cDNA was synthesized. The comparative cycle threshold (CT) approach was used to quantify gene expression, and the GAPDH gene was used as an internal reference. The experiment was repeated three times. (C) The expression of the CMP1 gene under nutrient or starvation condition. Overnight cultures of the H99 strain were washed and transferred to new YPD, YNB, MM, and DME medium and culture for 2 and $4 \mathrm{~h}$. Relative qRT-PCR was used to detect the expression of CMP1 under nutrient or starvation conditions, as described in (B). ns: not significant; ${ }^{* *}, p<0.01 ;{ }^{* * * *}, p<0.0001$.

\subsection{Cmp1 Regulates Capsule Formation}

To identify the role of Cmp1 in C. neoformans, we created the CMP1 gene disruption mutants cmp $1 \Delta$ in the two wild-type strains backgrounds (Figure 4A-D). The cmp1 $\triangle$ mutants complemented strain $c m p 1 \triangle:: C M P 1$ (TBL177 and TBL178, Figure 4E-G) and CMP1 overexpressed strains $C M P 1^{O E}$ (TBL186 and TBL187) were also generated. The complementation or overexpression of the CMP1 gene was also verified by qRT-PCR (Figures $4 \mathrm{G}$ and $5 \mathrm{~B}$ ). Since $\mathrm{Cmp} 1$ is a putative mannoprotein that may play a part in capsule formation, we first examined the role of $\mathrm{Cmp} 1$ in capsule formation, melanin production, and growth at $37^{\circ} \mathrm{C}$ in vitro. The $c m p 1 \triangle$ mutants and $C M P 1^{O E}$ strains generated normal melanin on Niger-seed agar plates and grew normally at $37^{\circ} \mathrm{C}$ (Figures $5 \mathrm{~A}$ and 6) compared to that of the $\mathrm{H} 99$ strain. However, both the $\operatorname{cmp} 1 \triangle$ mutants and CMP1 overexpressed strains exhibit reduced capsule size when cultured in DME, MM, and RPMI (Roswell Park Memorial Institute 1460)-containing medium. Based on a Student's $t$-test, the difference of the capsule size between the two strains and the H99 strain was significant, which indicated that $\mathrm{Cmp} 1$ is vital for the development of capsule in vitro (Figure 5C-E).

Then we investigated the $\operatorname{cmp} 1 \triangle$ mutants and $C M P 1^{\mathrm{OE}}$ strains grown in various stresses, including starvation stress (YNB), high-temperature stress $\left(37^{\circ} \mathrm{C}\right)$, cell wall stress $(0.5 \%$ Congo red or $0.025 \%$ SDS), osmotic stress (1.5 M Sorbitol or $1.5 \mathrm{M} \mathrm{NaCl})$, oxidative stress $\left(2.5 \mathrm{mM} \mathrm{H}_{2} \mathrm{O}_{2}\right)$, nitrosative stress $\left(1 \mathrm{mM} \mathrm{NaNO}_{2}, \mathrm{pH}=4.0\right)$, and drugs (Hyg and $\left.\mathrm{HU}\right)$. Our results revealed that under the above stresses, there was no difference between the growth of cmp $1 \triangle$ mutants and the CMP1 overexpressed strains compared with the wild-type strains, indicating that $\mathrm{Cmp} 1$ did not participate in response to the above stress conditions (Figure 6). 


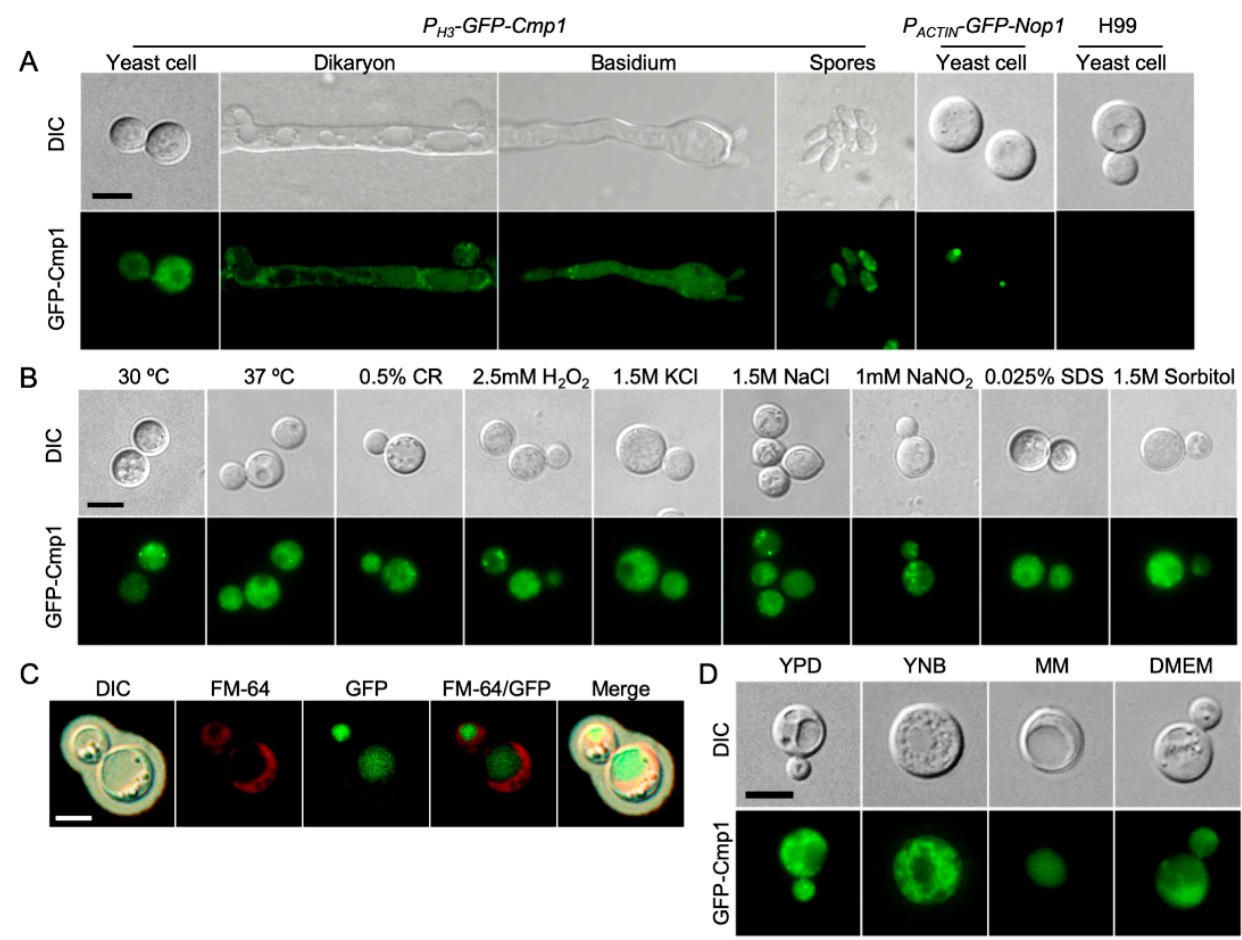

Figure 3. Subcellular localization of Cryptococcus Cmp1. (A) The yeast cells, dikaryon, basidium, and basidiospores of the strain expressing GFP-Cmp1 were observed by confocal microscopy (Olympus, FV1200). The fusion protein of GFP and Cryptococcus Cmp1 (GFP-Cmp1) is localized in the cytoplasm of $C$. neoformans. Yeast cells of the nuclear-localized GFP-Nop1 strain and the wild-type H99 strain were positive and negative controls. Scale bar, $5 \mu \mathrm{m}$. (B) GFP-Cmp1's localization in yeast cells under different stresses. CR: Congo red. Scale bar, $5 \mu \mathrm{m}$. (C) GFP-Cmp1's localization in yeast cells after cultured in MM medium for three days. (D) GFP-Cmp1's localization in yeast cells after cultured in capsule-inducing media for three days. Scale bar, $5 \mu \mathrm{m}$.

\subsection{Cmp1 is not Required for Sexual Reproduction}

C. neoformans is a heterothallic basidiomycete having two mating types: MATa and MAT $\alpha$. The haploid cells of two opposite mating types can fuse and undergo sexual reproduction, generating the dikaryotic mating hyphae and the haploid basidiospores. To confirm whether $\mathrm{Cmp} 1$ functions in cryptococcal sexual reproduction, we generated the cmp1 $\triangle$ mutants and CMP1 overexpressed strains in both $\mathrm{H} 99$ and KN99a strains. The mating filaments and basidiospores production was investigated in both $c m p 1 \Delta$ mutants bilateral mating $(\operatorname{cmp} 1 \Delta \times c m p 1 \Delta)$ and unilateral mating ( $c m p 1 \Delta \times$ wild type). However, no noticeable phenotypic changes were observed in both $c m p 1 \Delta$ bilateral or unilateral-mating assays than the wild-type strains (Figure 7). Additionally, the $C M P 1^{O E}$ strains generated normal mating filaments and basidiospores in bilateral mating (Figure 7). These data suggest that Cmp1 is not necessary in the sexual reproduction of $C$. neoformans.

\subsection{Cmp1 is Required for Fungal Infection}

Since Cmp1 regulates the formation of the capsule of $C$. neoformans, we speculated that Cmp1 might play a part in the fungal virulence of $C$. neoformans. To examine our prediction, we explored the virulence of $c m p 1 \triangle$ mutant and $C M P 1^{O E}$ strain in a murine inhalation model of cryptococcosis. Ten female C57 BL/6 mice in each group were infected intranasally with $10^{5}$ cells of each cryptococcal strain, and the mice were examined twice a day. Consistent with our previous results, all the mice inoculated with the wild-type strain were terminated with a lethal infection between 18 and $25 \mathrm{dpi}$. By contrast, the $\operatorname{cmp} 1 \Delta$ mutant's virulence was attenuated significantly, and five of the cmp $1 \Delta$ mutants-infected mice survived at the endpoint of the animal experiment ( $80 \mathrm{dpi}$; Figure $8 \mathrm{~A}$ ), which was remarkably 
different from that of wild-type control ( $p<0.0001$, log-rank (Mantel-Cox) test). The cmp1 $\Delta$ mutant complemented strains developed a lethal infection in mice approximately $21-29 \mathrm{dpi}$, which exhibited no marked difference with the H99 strain ( $p>0.9999$, log-rank (Mantel-Cox) test), confirming that the attenuation of virulence in the cmp $1 \triangle$ mutant was caused by the disruption of the CMP1 gene (Figure $8 \mathrm{~A}$ ). Interestingly, the $C M P 1^{O E}$ strains also showed significant virulence defect compared with the wild-type H99 strain, $(p<0.0001$, log-rank (Mantel-Cox) test; Figure 8A).
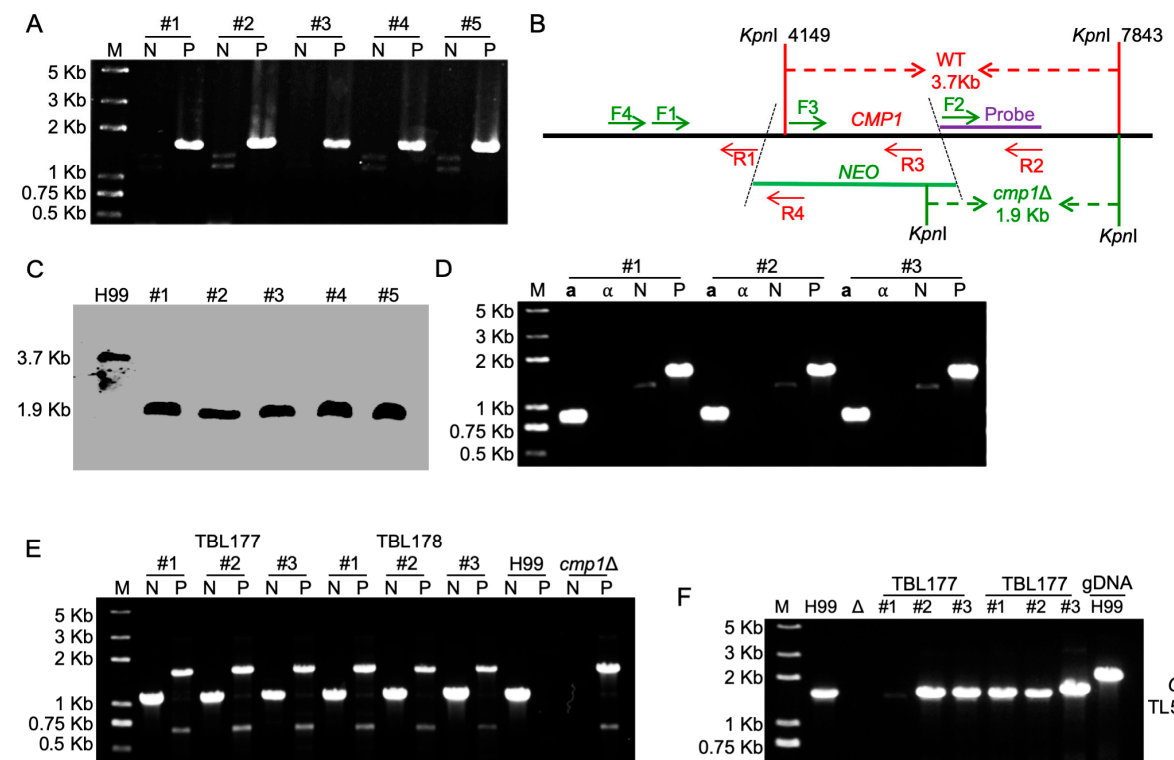

G
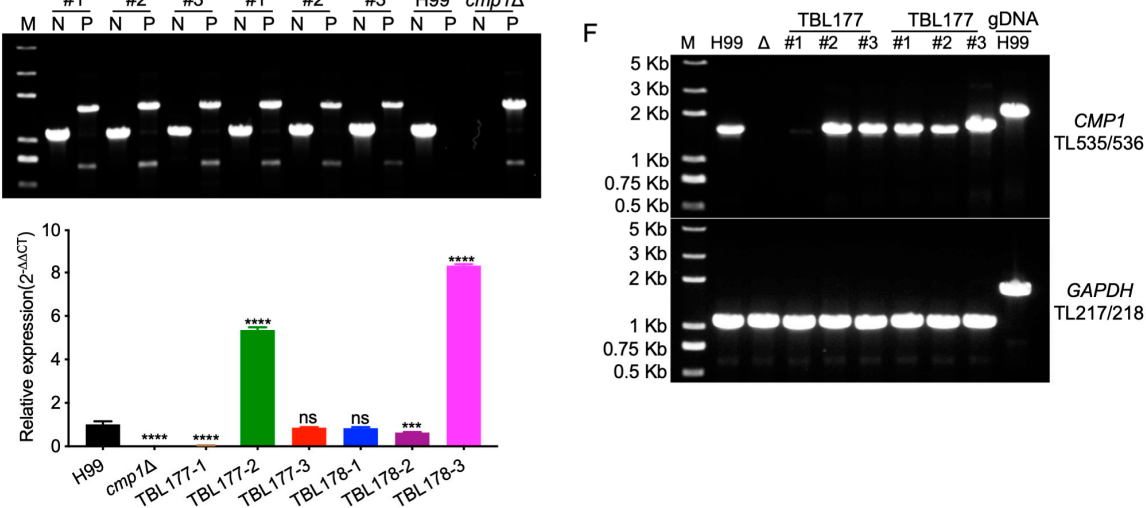

Figure 4. Generation and confirmation of CMP1 gene knockout, complementation, and overexpression strains. (A) The diagnostic PCR confirmation of the five transformants with G418 resistance. P: primers for positive amplification, TL241/TL59 (F4/R4 in 3B); N: primers for negative amplification, TL239/TL240 (F3/R3 in 3B). (B) Restriction enzyme used in the digestion of the genomic DNAs in Southern blotting. The probe was synthesized using the TL237/TL238 (F2/R2) PCR products as templates. The cmp1s mutants will produce a $1.9 \mathrm{~Kb}$ band while the wild-type strain $\mathrm{H} 99$ generates a $3.7 \mathrm{~Kb}$ band. (C) Southern blotting analysis of the CMP1 knockout mutants. An equal amount of genomic DNA (50 $\mu \mathrm{g})$ was digested with $\mathrm{KpnI}$, fractionated, and hybridized with a CMP1 downstream flanking sequence-specific probe, as shown in Figure 3B. As anticipated, a 1.9 and $3.7 \mathrm{~Kb}$ bands were generated in the cmp $1 \Delta$ mutants and the wild-type strain $\mathrm{H} 99$, respectively. (D) Verification of the a mating-type $\mathrm{cmp} 1 \Delta$ mutants. a: a mating type-specific primers, TL69/70; $\alpha$ : $\alpha$ mating type-specific primers, TL67/68; N: primers for negative amplification, TL239/TL240 (F3/R3 in 3B); P: primers for positive amplification, TL241/TL59 (F4/R4 in 3B). (E-G) Generation and verification of CMP1 complementation strains. The genome DNA (E) and cDNA (F) of the complementation transformants were used as templates for PCR amplification to verify the CMP1 gene's complementation. N: primers for negative amplification, TL239/TL240 (F3/R3 in 4B); P: primers for positive amplification, TL241/TL59 (F4/R4 in 4B); the expression of CMP1 of the complementation transformants was measured by relative qRT-PCR. ns: not significant; ${ }^{* * *}, p<0.001$; ****, $p<0.0001$. 

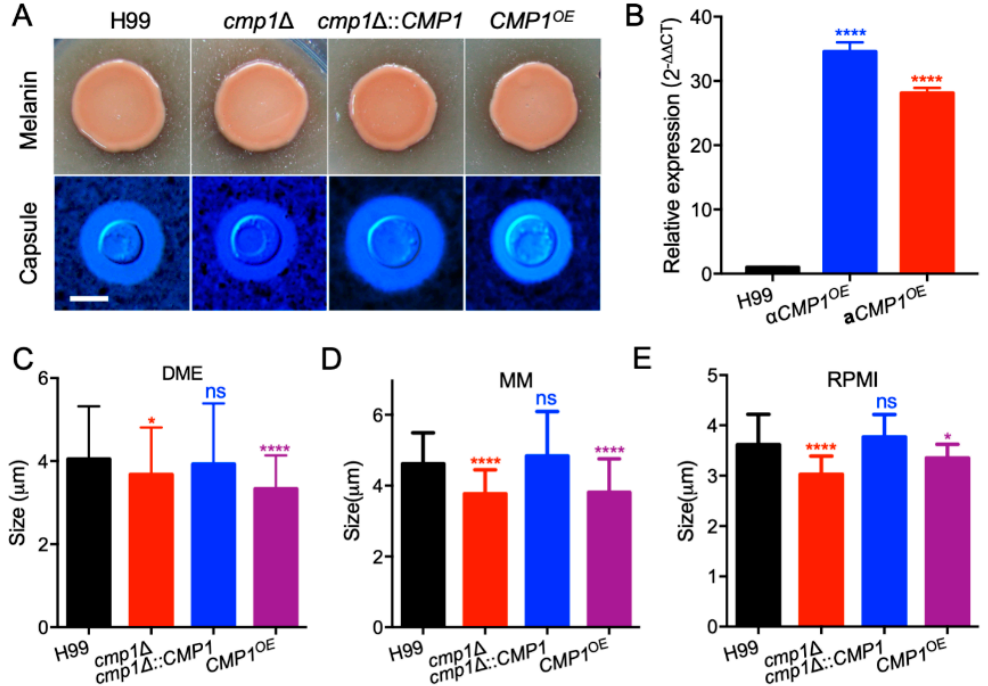

Figure 5. Cmp1 is necessary for Cryptococcus capsule formation. (A) Melanin formation (top) and capsule production (bottom) were carried out on Niger seed medium and DME medium, respectively. The melanin levels generated by the Cryptococcus strains were photographed after incubation at $37^{\circ} \mathrm{C}$ for two days. The Cryptococcus cells were cultured in the DME medium at $37^{\circ} \mathrm{C}$ for three days, and the formation of the capsule was observed by India ink staining. Bars, $5 \mu \mathrm{m}$. (B) The overexpression of the CMP1 gene was evaluated by the relative qRT-PCR analysis. To compare the differences in gene expression, we arbitrarily set the expression levels of the CMP1 gene in H99 strain grown on YPD medium as 1. ${ }^{* * * *}, p<0.0001$. (C-E) Statistical analysis of cryptococcal capsule production in DME, MM, and RPMI-containing medium. The capsule size from at least 100 cells was measured, and the data shown are the average with standard deviation from three repeats. ns: not significant; ${ }^{*}, p<0.05$; $* * * *, p<0.0001$.

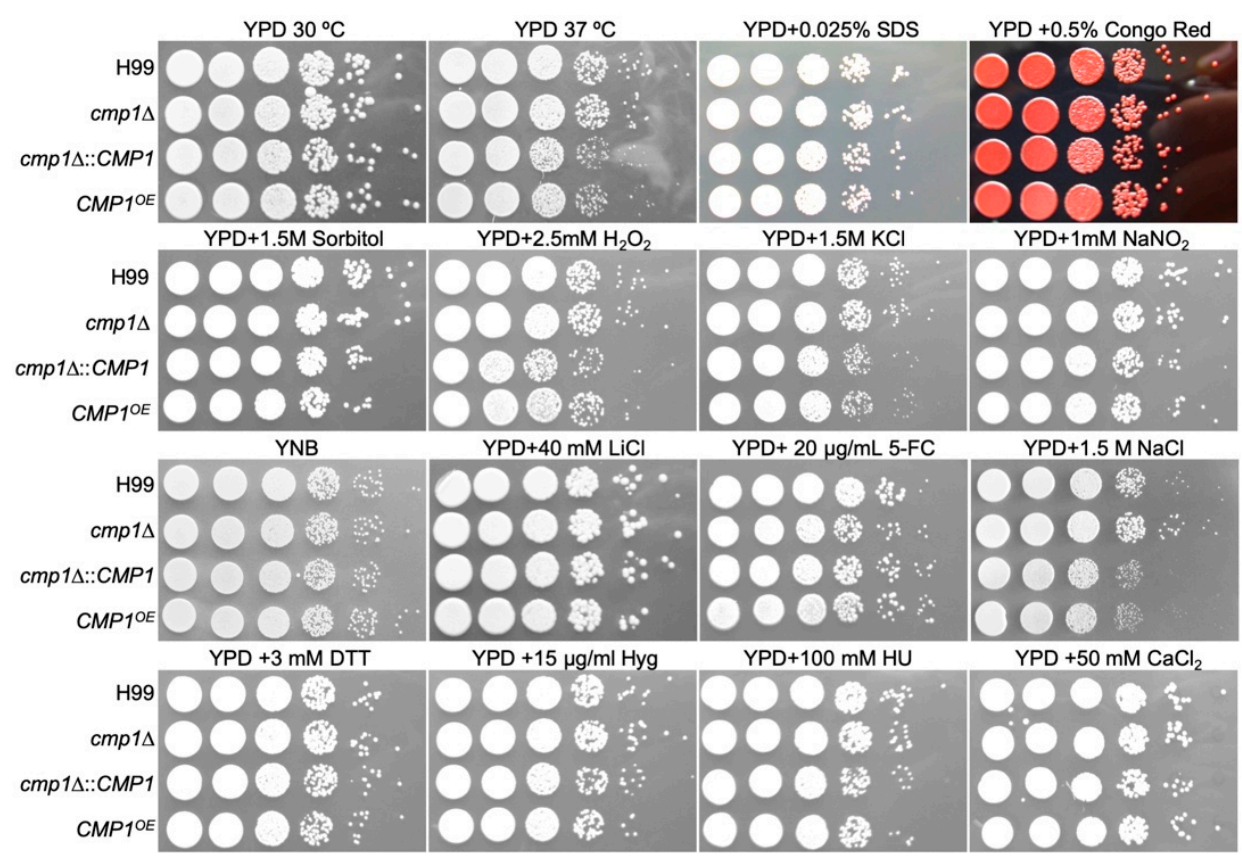

Figure 6. Growth of cmp1 $1 \Delta$ mutants under different stress conditions. Each strain's overnight culture was first diluted to an OD600 value of 2.0, and then ten-fold serial dilutions were prepared with $\mathrm{ddH}_{2} \mathrm{O}$. Of each dilution $5 \mu \mathrm{L}$ were dropped on YPD plates with different stresses and then incubated at $30{ }^{\circ} \mathrm{C}$ for 2 or 3 days. The culture conditions are shown at the top, and the Cryptococcus strains at the left. 5-FC: 5-fluorocytosine; DTT: dithiothreitol; HU: hydroxyurea. 


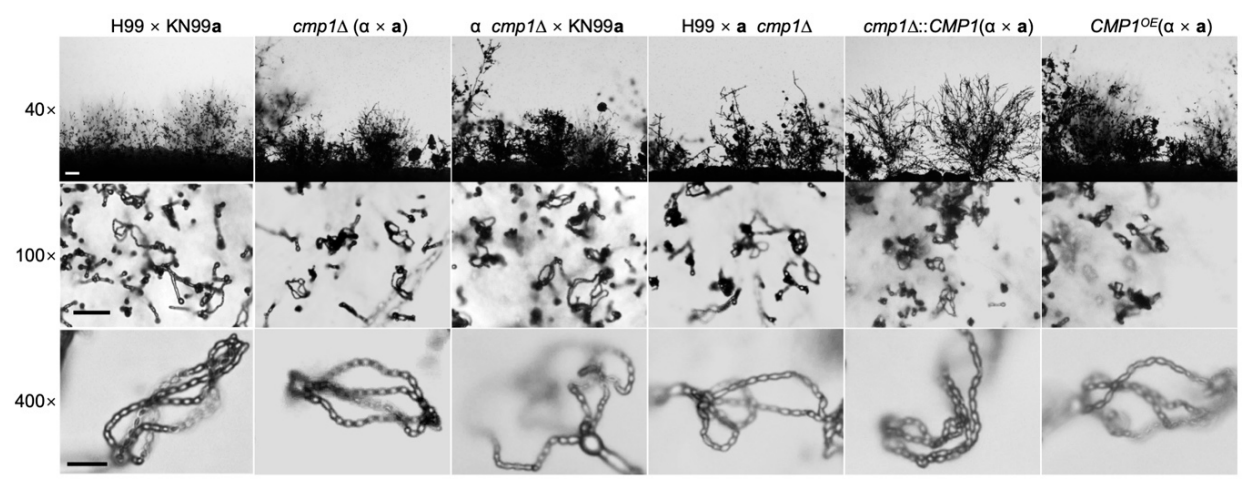

Figure 7. Deletion or overexpression of CMP1 shows no effect on filamentation and sporulation during mating. Bilateral and unilateral mating assays were carried out on MS medium with the wild-type, cmp $1 \Delta$ mutants, cmp1 1 complemented strains, and CMP1 overexpression strains. After two weeks of incubation at $25^{\circ} \mathrm{C}$ in the dark, images of the mating structures were captured at $\times 40$ magnification (top, bar $=100 \mu \mathrm{m}$ ), $\times 100$ magnification (middle, bar $=50 \mu \mathrm{m}$ ), and $\times 400$ magnification (bottom, bar $=10 \mu \mathrm{m})$.

A
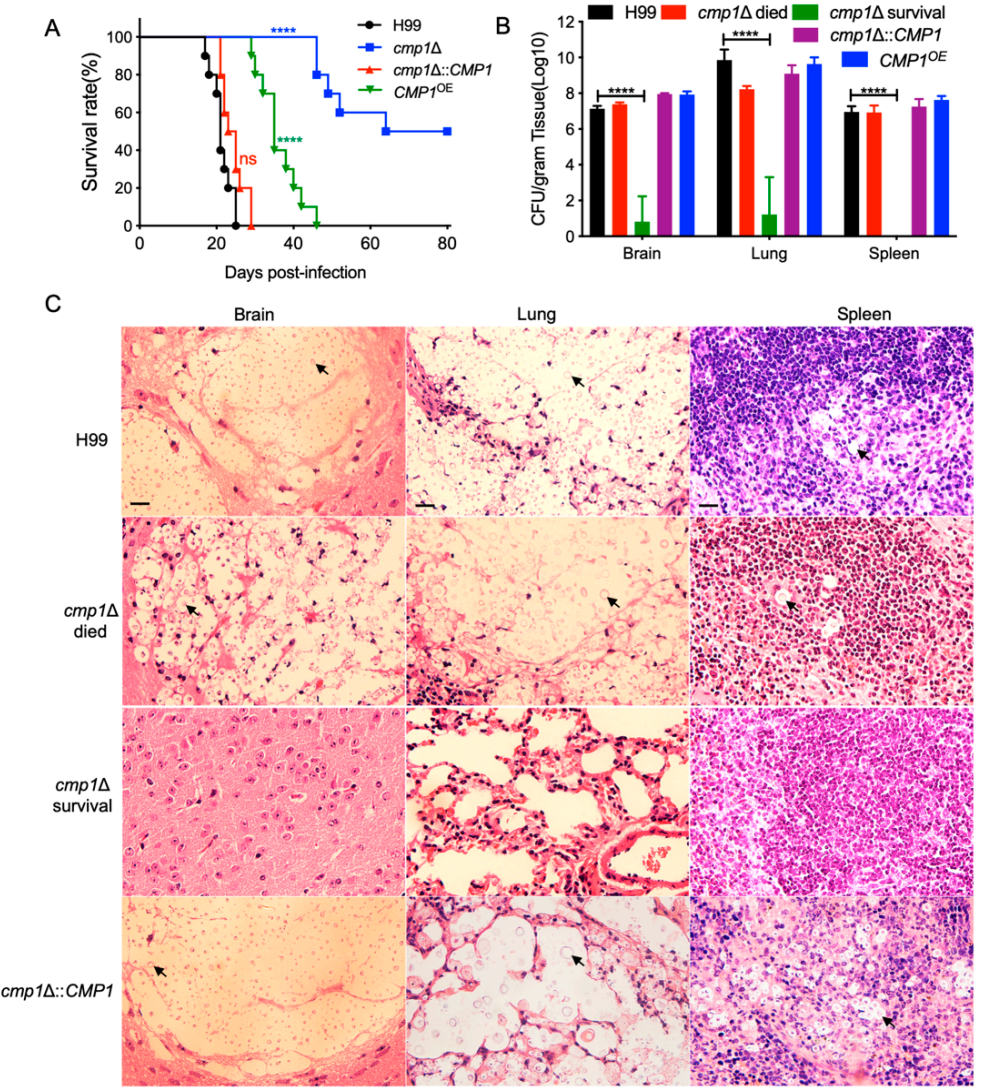

Figure 8. Cmp1 is required for full virulence of $C$. neoformans. Ten C57 BL/6 mice in each group were infected intranasally with $10^{5}$ cells of the wild-type H99, the cmp1 $1 \Delta$ mutant, the cmp $1 \Delta:: C M P 1$ complemented strain, and the $C M P 1^{\mathrm{OE}}$ strain. (A) Survival rates of $\mathrm{C} 57 \mathrm{BL} / 6$ mice after infection. Both the $\operatorname{cmp} 1 \Delta$ mutant and the $C M P 1^{\mathrm{OE}}$ strain are less virulent than the wild-type $\mathrm{H} 99$ strain. ns: not significant; ****, $p \leq 0.0001$ (determined by the log rank (Mantel-Cox) test). (B) Fungal load (CFU) in brains, lungs, and spleens. The data shown are the mean \pm SD for the values of five mice. ****, $p \leq 0.0001$ (determined by Mann-Whitney test). (C) Hematoxylin-eosin stained slides from the organs' cross-sections at the end of the infection experiment were prepared and observed under a light microscope. Arrows indicate the cryptococcal cells. Scale bar, $20 \mu \mathrm{m}$. 
To find out why was the virulence of the $c m p 1 \Delta$ mutant and the $C M P 1^{\mathrm{OE}}$ strain attenuated, we examined the fungal load of the infected mice when the infection experiment was terminated. The brains, lungs, and spleens of five mice infected with each cryptococcal strain were dissected, and the fungal load of these organs was assessed as yeast CFU per gram fresh organs. Our results indicated that $10^{7}, 10^{9}$, and $10^{7} \mathrm{CFU}$ were recovered from the brains, lungs, and spleens at the endpoint of the infection experiments, respectively when the mice inoculated with the wild-type H99 strain (Figure 8B). Organs of the mice infected by the cmp $1 \Delta$ mutants were also dissected to calculate the fungal burdens. From the brains, lungs, and spleens of the mice sacrificed before $80 \mathrm{dpi}, 10^{7}, 10^{8}$, and $10^{7} \mathrm{CFU}$ were recovered. In contrast, only about $10^{2}$ and $10^{3} \mathrm{CFU}$ were isolated from the brain and lung, and no cryptococcal cells were found from the spleens of the mice sacrificed at $80 \mathrm{dpi}$. Organs of the mice infected by the $C M P 1^{\mathrm{OE}}$ strains were also dissected to calculate the fungal burden, and $10^{7}, 10^{9}$, and $10^{7}$ $\mathrm{CFU}$ were isolated from the brains, lungs, and spleens, respectively (Figure 8B).

Additionally, hematoxylin-eosin stained slides were prepared to visualize the development of fungal lesions in the organs of mice infected by Cryptococcus. As shown in Figure 8C, infection by both the wild-type strain and the cmp1 $:: C M P 1$ complemented strain resulted in severe damage in mouse brains at the infection experiment's endpoint. However, it took more than 50 days for the cmp $1 \Delta$ mutant to cause similar damage, showing that the $c m p 1 \Delta$ mutant's virulence was significantly attenuated. By contrast, the cmp $1 \Delta$ mutants can only cause very little damage to the infected brain of the mice sacrificed at $80 \mathrm{dpi}$, indicating that the $\operatorname{cmp} 1 \Delta$ mutants' virulence was significantly attenuated.

Meanwhile, in the lungs infected by the H99 or the $\operatorname{cmp} 1 \triangle:: C M P 1$ complemented strain at $23 \mathrm{dpi}$, and cmp $1 \Delta$ mutants at $50 \mathrm{dpi}$, large numbers of cryptococcal cells with thickened capsules can be visualized to cause severe damage to the organs. However, no detectable damage was visualized in the lungs of the $c m p 1 \Delta$-infected mice that survived even at $80 \mathrm{dpi}$ (Figure $8 \mathrm{C}$ ). Similar results were also observed in the spleens infected by the above cryptococcal strains (Figure 8C). These data suggest that the Cmp1 is indispensable for the development of cryptococcosis in a mouse model.

To find out why the cmp $1 \Delta$ mutants are virulence defective, we examined the fungal loads in infected mice's organs at 7,14 , and $21 \mathrm{dpi}$. CFU counts results show that the cmp $1 \Delta$ mutant could still cause organ infection in the infected mice, but the CFUs recovered from organs of the mice infected by the cmp $1 \Delta$ mutant were significantly lower than that of the wild-type H99 strain (Figure 9A-C). Histopathologic findings also indicated that the cmp1 $1 \Delta$ mutants could still cause lung infections, but compared with the wild type or the cmp $1 \triangle:: C M P 1$ complemented strains infected mice, the cell density was smaller and lesion development happened later and was less extensive (Figure 9D, and data not shown).

\subsection{Cmp1 is Important for Proliferation Inside Macrophage and Survival in the Host Complement System}

Our virulence study showed that the $\operatorname{cmp} 1 \Delta$ mutant's virulence was significantly attenuated in a mouse systematic inhalation model. Since macrophages and monocytes are the first host cells that C. neoformans encountered after it gets into the host lung, we hypothesized that the cmp $1 \Delta$ mutant might have proliferation defects in host macrophages, and it is difficult for extracellular cryptococcal cells to survive in the hostile environment inside the host. To test our hypotheses, we first carried out a Cryptococcus-macrophage interaction assay using the RAW246.7 murine macrophage cells. After two hours of coincubation of the cryptococcal cells and macrophages, the yeast CFU number recovered from the cmp $1 \Delta$ mutant-coincubated macrophages was comparable to that recovered from the macrophages coincubated with wild-type strains, indicating a similar phagocytosis level between the cmp $1 \Delta$ mutant and the wild-type strains (Figure 10A). 
A
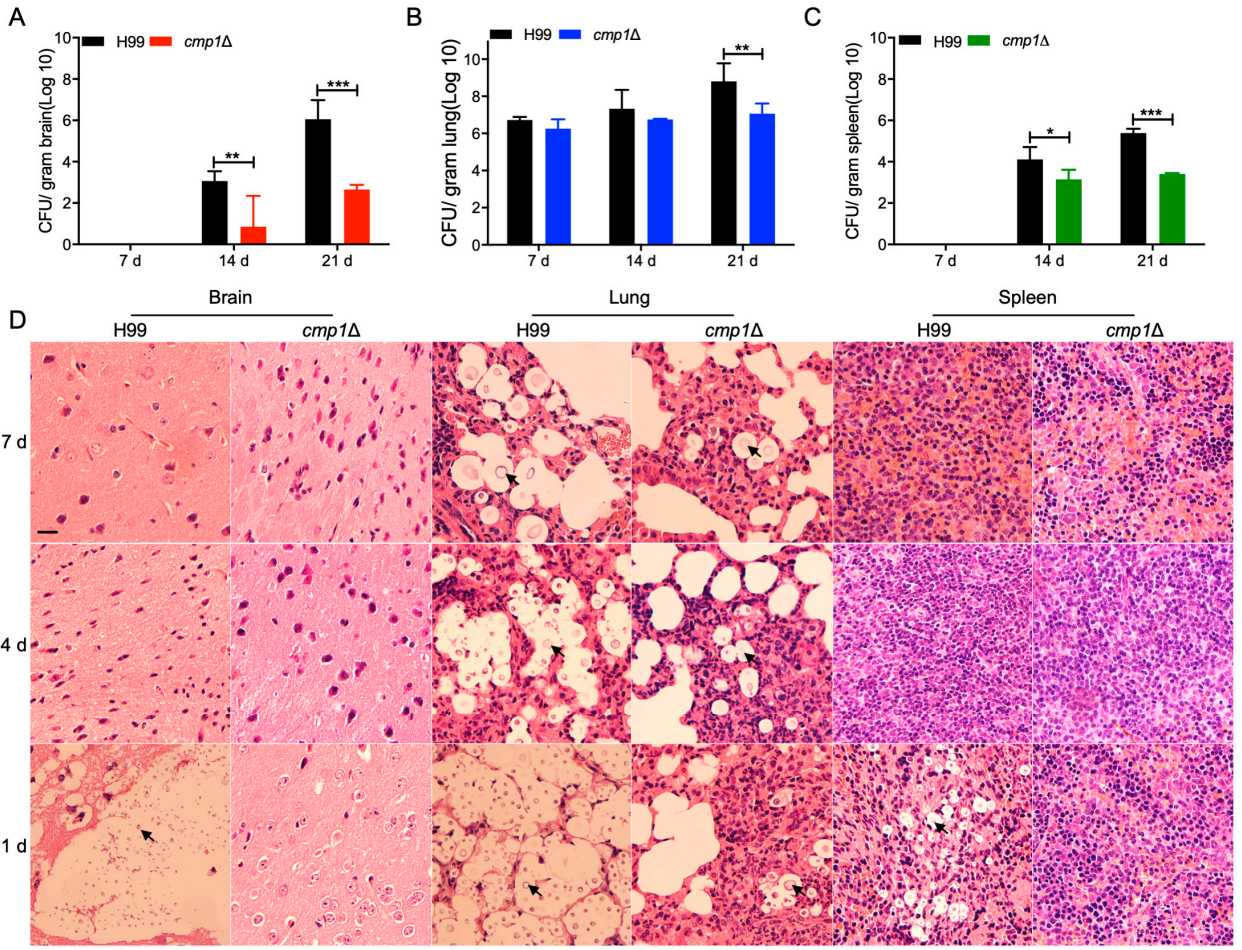

B

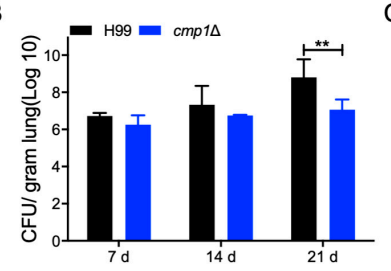

C

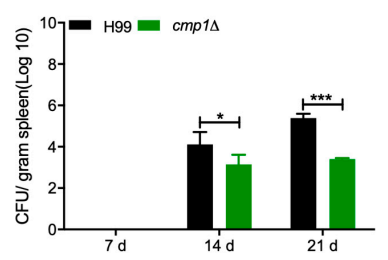

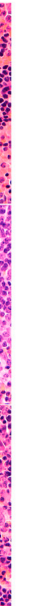

Figure 9. Progression of $c m p 1 \Delta$ mutant infection in vivo. Organs from mice inoculated with $\mathrm{H} 99$ or the cmp1 $1 \Delta$ mutant were dissected at 7, 14, and $21 \mathrm{dpi}$. CFUs were calculated in the homogenates of the brains (A), lungs (B), and spleens (C). The data shown are the mean \pm SD for values from five mice. ${ }^{*}, p \leq 0.05 ;{ }^{* *}, p \leq 0.01 ;{ }^{* *}, p \leq 0.001$ (determined by the Mann-Whitney test). (D) Hematoxylin-eosin stained slides from the cross-sections of the brains, lungs, and spleens were also prepared and observed by light microscopy. Arrows indicate the cryptococci. Bar, $20 \mu \mathrm{m}$.
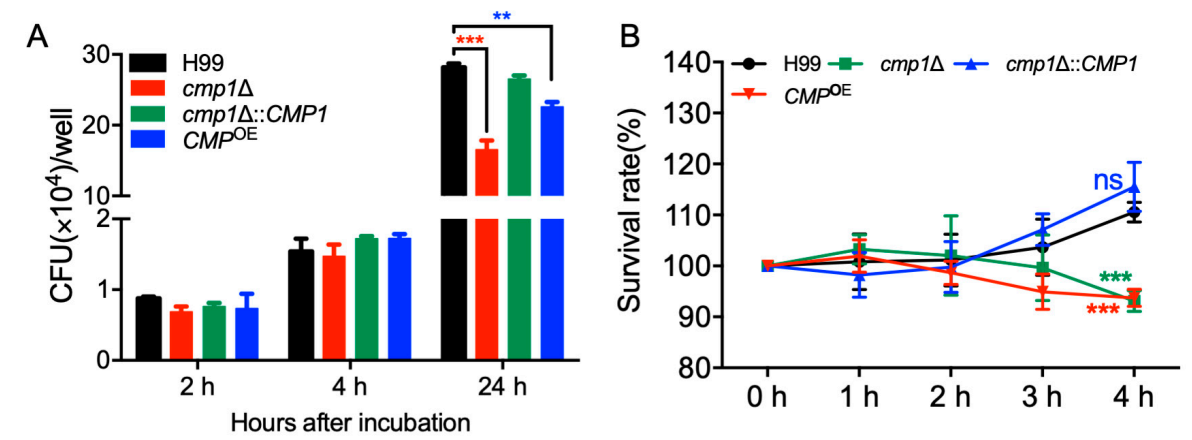

Figure 10. Cmp1 is important for proliferation inside macrophage and survival in the host complement system. (A) Cryptococcus' proliferation inside macrophage was carried out using the RAW246.7 murine macrophage cells. PBS-washed Cryptococcus strains (H99, cmp1 $1 \Delta$ mutant, cmp1 $:: C M P 1$ and $C M P 1{ }^{\mathrm{OE}}$ ) were coincubated with activated macrophages for $2 \mathrm{~h}$. The cells were incubated for an additional 0,2 , or $22 \mathrm{~h}$ after removing the nonadherent extracellular Cryptococcus cells by washing with fresh DMEM and then lysed by $\mathrm{H}_{2} \mathrm{O}$ for $30 \mathrm{~min}$. The lysate was dispersed on the YPD plates, and the intracellular proliferation and macrophage killing were determined by CFU counting. ${ }^{* *}, p<0.01 ;{ }^{* * *}, p<0.001$. (B) Overnight cultures of the same strains as in (A) were washed twice with PBS buffer and diluted to a final concentration of $1 \times 10^{7} \mathrm{CFU} / \mathrm{mL}$. Fifty microliters of each strain's cell suspensions were added to 450 microliters of mouse serum and incubated at $37^{\circ} \mathrm{C}$ for indicated times. The precooled PBS buffer was added to stop the reaction, and the cells were washed twice with PBS buffer. One hundred microliters of the dilute $\left(10^{3}\right.$ dilution) was spread on YPD plates, and the number of CFU was used to measure the Cryptococcus cell viability. ns: not significant; ${ }^{* * *}, p<0.001$. 
However, after $24 \mathrm{~h}$ of incubation, the yeast CFU recovered from the cmp $1 \Delta$ mutant-interacting macrophages was significantly fewer than that of the wild-type-interacting macrophages $(p<0.001$, Figure 10A). These results suggested that the $\operatorname{cmp} 1 \Delta$ mutant proliferates slower than the wild-type strain once it is engulfed by macrophages, which could be one reason why the cmp $1 \Delta$ mutants have significant virulence attenuation in the mouse systemic infection model.

Meanwhile, we also tested the Cryptococcus cells' viability after incubation with mouse serum for $1,2,3$, and $4 \mathrm{~h}$ to verify whether components of the host complement system damage C. neoformans. Our results showed that the survival rate of $\operatorname{cmp} 1 \Delta$ mutants was significantly lower than that of the wild-type strains after $4 \mathrm{~h}$ incubation of Cryptococcus cells with the mouse serum $(p<0.001$, Figure 10B), which also indicated that components of the host complement system did have more severe damage on $c m p 1 \Delta$ mutants.

The above results indicated that the disruption of the CMP1 gene might affect Cryptococcus' virulence by affecting the proliferation inside macrophages or affecting the viability of Cryptococcus in the host complement system.

\section{Discussion}

Despite its low content, mannoprotein is a crucial component of the Cryptococcus polysaccharide capsule, stimulating the host's immune protection. In this study, we identified and functionally analyzed a predicted mannoprotein $\mathrm{Cmp} 1$, and our results showed that the $\mathrm{Cmp} 1$ is a predicted mannoprotein and is required for fungal virulence in $C$. neoformans.

In the literature, at least four mannoproteins (MP84, MP88, MP98, and MP115) in C. neoformans have been suggested to play a part in stimulating host T-cell responses $[10,14,19,33]$. The fifth mannoprotein, Cig1, has been proved to help increase the virulence of $C$. neoformans by supporting iron acquisition from heme [21]. Among these five mannoproteins, only Cig1 was tested and shown to have virulence properties in mouse models. In our previous work identifying the downstream targets of the F-box protein $\mathrm{Fbp} 1$, we found that $\mathrm{Cmp} 1$ might be a downstream substrate of Fbp1 due to its high abundance in fbp $1 \Delta$ mutant background (Table 1 ). However, when we did a sequence analysis of $\mathrm{Cmp} 1$, we found that $\mathrm{Cmp} 1$ did not have the signal peptide sequence typical of mannose glycoproteins (Figure 1C). Unlike other mannoproteins, e.g., MP98, located near the cell wall [9], subcellular localization indicates that Cmp1 is located in the cytoplasm. More interestingly, however, when we examined the subcellular localization of Cmp1 under nutrient or starvation conditions, we found that the localization of Cmp1 differs depending on the culture conditions. When cultured in nutrient media such as YPD, YNB, and DMEM, the Cmp1 proteins localized in the cytoplasm of the Cryptococcus cells. However, the Cmp1 proteins is localized in the vacuoles when cultured in the capsule-inducing medium, e.g., MM, which might be because the capsule-inducing medium is a nutrient-deficient medium, and Cryptococcus cells cannot get enough nutrients from it and has to degrade its own substances, which causes the GFP-cmp1 protein to accumulate in the vacuole. Our results suggest that $\mathrm{Cmp} 1 \mathrm{might}$ be a mannoprotein that does not contain a signal peptide and cannot be secreted outside the cell.

In the opportunistic dimorphic fungus Talaromyces marneffei, Mp1p, and its $13 \mathrm{Mp} 1 \mathrm{p}$ homologs (MpLp1-13) were identified in its genome [26]. Among the $13 \mathrm{Mp} 1 \mathrm{p}$ homologs, the MpLp7 has no signal peptide sequence, while the remaining $12 \mathrm{Mp} 1 \mathrm{p}$ homologues contain putative signal peptides, varying amounts of putative O- and N-glycosylation sites, or glycosylphosphatidylinositol (GPI) anchors [26]. The presence of mannoprotein without a signal peptide in T. marneffei is also suggested that the Cryptococcus $\mathrm{Cmp} 1$ might be a mannoprotein without a signal peptide. Meanwhile, our results also showed that the CMP1 gene knockout or overexpression could affect the capsule's formation, one of the three virulence factors. However, since $\mathrm{Cmp} 1$ is an intracellular localized protein and is located in vacuoles under the conditions that induce capsule formation, how $\mathrm{Cmp} 1$ affects Cryptococcus capsule formation remains unknown. Thus, further research is needed to determine how Cmp1 affects cryptococcal capsule formation. 
The molecular mechanism of Cmp1 regulating virulence is unclear and remains to be determined. Fungal virulence assay in this study showed that both disruption and overexpression of CMP1 resulted in significant virulence attenuation in $C$. neoformans (Figure 8). Progression of $c m p 1 \Delta$ mutant infection in vivo at 7,14 , and $21 \mathrm{dpi}$ indicated that while the cmp $1 \Delta$ mutant could still cause infection in infected organs, the cell density was smaller and lesion developed later and was less extensive compared with the H99 infected mice (Figure 9). However, these data cannot explain the molecular mechanism by which Cmp1 controls the virulence of $C$. neoformans. Disruption or overexpression of CMP1 affects capsule formation in C. neoformans, but whether this directly affects Cryptococcus virulence is still unknown.

Macrophages are the key defensive cells against Cryptococcus infection. In our study, the cmp $1 \Delta$ mutant's virulence was significantly attenuated in a mouse systematic infection model. Since macrophages or monocytes are the first host cells that $C$. neoformans encountered after it gets into the host lung, we hypothesized that the $\operatorname{cmp} 1 \Delta$ mutant might have proliferation defects in host macrophages. Our Cryptococcus-macrophage interaction assay did show that the cmp $1 \Delta$ mutant proliferates slower than the wild-type strain once it is engulfed by macrophages (Figure 10A). Besides, the Cryptococcus cells' viability assay in our study showed that the survival rate of $c m p 1 \Delta$ mutants was significantly lower than that of the wild-type strains after incubation with mouse serum (Figure 10B). Both of the above two experimental results showed that disruption of the CMP1 gene significantly affect the proliferation or survival of Cryptococcus cells in the host, which could be one reason why the cmp1 $\Delta$ mutants have a significant virulence attenuation in the mouse systemic infection model.

Fungal pathogens, such as C. neoformans, A. fumigatus, C. albicans, and T. marneffei are highly virulent fungi, causing high fatalities in HIV-positive patients, transplant recipients, and patients receiving corticosteroid therapy [34,35]. However, there are relatively few antifungal drugs currently, and the latest antifungal drugs have shown resistance in these pathogens. The fungal cell wall made up of $\alpha$ and $\beta$ glucans, chitin, chitosan, and mannoproteins, is vital for fungal growth and development and is also one of the most significant drug development targets [36,37]. The results of previous studies showed that mannoproteins play a vital role in fungal virulence or cell wall integrity, such as Cig1 in C. neoformans, Krp1 in C. gattii, and Mp1p in T. marneffei [21,22,26]. Given mannoproteins' role in fungal virulence or cell wall integrity in fungal pathogens, it might be one of the potential targets for antifungal drug development.

\section{Materials and Methods}

\subsection{Ethics Statement}

The animal experiments conducted at the Southwest University were in complete accordance with the Ministry of Science and Technology of China's "Guidelines for the Ethical Care of Laboratory Animals (No. 398, 2006)". Southwest University's Animal Ethics Committee approved all vertebrate experiments.

\subsection{Strains and Growth Conditions}

Cryptococcus strains used in the present study are shown in Table 2. The wild-type strains of Cryptococcus $\mathrm{H} 99$ and KN99a were routinely preserved in our laboratory. All other cryptococcal strains were derived from wild-type strains. Cryptococcal strains are grown on YPD medium at $30^{\circ} \mathrm{C}$ or indicated temperatures. MS or V8 medium were used for induction of mating and prepared as described previously [38]. DME medium, minimal medium (MM), and RPMI-contaning medium were used for the induction of Cryptococcus capsule formation [39-41]. All other media used in this study were prepared as previously described [39]. 


\subsection{CMP1 Expression Pattern Assay}

To investigate the temporal expression CMP1, we amplified a $1738 \mathrm{bp}$ BamHI/BamHI promoter fragment of the CMP1 gene using the wild-type genomic DNA as templates with primers TL481/482 and inserted into the plasmid pTBL3 [31] to construct the CMP1 promoter and mCherry fusion vector pTBL82 (see Table 3 for plasmid information). To explore the cellular location of cryptococcal Cmp1 protein, we amplified the coding region of the CMP1 from the wild-type genomic DNA using primers TL554/555 and inserted into plasmid pCN19 to construct the GFP-CMP1 fusion expression vector, as previously described [31]. The resulting plasmids, pTBL82, and pTBL92 were linearized with $S c a I$ and XmnI, respectively, and biolistically transformed into the wild-type strains, H99 and KN99a, as described previously [31,42]. Stable transformants were further confirmed on the YPD containing $100 \mathrm{mg} /$ liter nourseothricin sulfate. The transformants' fluorescence was visualized by confocal microscopy (Olympus, FV1200).

Table 3. The primers used for PCR amplification in this study.

\begin{tabular}{|c|c|c|}
\hline Primers & Targeted Genes & Sequence $\left(5^{\prime}-3^{\prime}\right)$ \\
\hline TL17 & M13F & GTAAAACGACGGCCAG \\
\hline TL18 & M13R & CAGGAAACAGCTATGAC \\
\hline TL19 & NEO split F & GGGCGCCCGGTTCTTTTTGTCA \\
\hline TL20 & NEO split R & TTGGTGGTCGAATGGGCAGGTAGC \\
\hline TL59 & NEO R4 & TGTGGATGCTGGCGGAGGATA \\
\hline TL67 & STE20A $\alpha \mathrm{F}$ & CCAAAAGCTGATGCTGTGGA \\
\hline TL68 & STE20A a R & AGGACATCTATAGCAGAT \\
\hline TL69 & STE20A a F & TCCACTGGCAACCCTGCGAG \\
\hline TL70 & STE20A a R & ATCAGAGACAGAGGAGCAAGAC \\
\hline TL217 & GAPDH qRT-PCR F & TGAGAAGGACCCTGCCAACA \\
\hline TL218 & GAPDH qRT-PCR R & ACTCCGGCTTGTAGGCATCAA \\
\hline TL235 & CMP1 KO F1 & GGGGTAAAAGAGGGAGGATGAGAC \\
\hline TL236 & CMP1 KO R1 & CTGGCCGTCGTTTTACAGAACACCCGCCGCTGAACTTT \\
\hline TL237 & CMP1 KO F2 & GTCATAGCTGTTTCCTGCTCCGCTGCAACCAAGGCTACCA \\
\hline TL238 & CMP1 KO R2 & TGCGCGGCTCGAGACACAAGA \\
\hline TL239 & CMP1 KO F3 & TCCTCCGACTCGCGCCTCATCAG \\
\hline TL240 & CMP1 KO R3 & AGCTATCGCCGGCCCATTACCATC \\
\hline TL241 & CMP1 KO F4 & GCCCACGCGCCCACATACAT \\
\hline TL481 & CMP1 PRO F1 & ACGGTATCGATAAGCTTGACGGGTGCGGACGACATTTAGATTT (HindIII) \\
\hline TL482 & CMP1 PRO R1 & CTAGAACTAGTGGATCCACAGCATGCACACTCTCTGCAT (BamHI) \\
\hline TL483 & CMP1-mCherry F1 & TTAGTAAACTCGCCCAACATGTCTGGATCCATGGCTGGCAGGTGGCCGCTGC (BamHI) \\
\hline TL484 & CMP1-mCherry R1 & CTTGCTCACCATTCTAGAACTAGTGGATCCAAGCAAGACGGCAGCACCAAAC (BamHI) \\
\hline TL535 & CMP1 QPCR F1 & TCCTGGTATCTCCACCTCTTC \\
\hline TL536 & CMP1 QPCR R1 & CAAACAGCATGCCGACAAC \\
\hline TL554 & GFP-CMP1 F & GACGAGCTGTAcGGATCCATGGCTGGCAGGTGGCCGCTGCAC (BamHI) \\
\hline TL555 & GFP-CMP1 R & CTGGCGGCCGTTACTAGTTTAAAGCAAGACGGCAGCACCAAA (SpeI) \\
\hline TL562 & $C M P 1$ Comp F & GATATCGAATTCCTGCAGCCCGGGGGATCCGCCCACGCGCCCACATACATCCTCGC (BamHI) \\
\hline TL563 & CMP1 Comp R & CGGTGGCGGCCGCTCTAGAACTAGTGGATCCTGCGCGGCTCGAGACACAAGAGTAGA (BamHI) \\
\hline
\end{tabular}

Meanwhile, to detect the CMP1 gene expression during cryptococcal mating, we used qRT-PCR to detect the CMP1 gene's expression at mRNA levels. Mating cultures preparation, total RNAs isolation and purification, and cDNAs synthesis were carried out as described previously [31]. Gene expression of CMP1 was analyzed by the comparative $C_{\mathrm{T}}$ approach using the GAPDH gene as an internal control, as described previously [31].

\subsection{Generation of CMP1 Deletion, Complementation, and Overexpression Strains}

The CMP1 gene was disrupted in both wild-type strains using a split marker strategy, as described previously [31,43]. Primers TL235/TL236 and TL237/TL238 were used to amplify the $5^{\prime}$ and $3^{\prime}$ regions of the CMP1 gene, respectively, using $\mathrm{H} 99$ genomic DNA as templates (for primer sequences, see Table 2). The M13 primers (TL17 and TL18) were used to amplify the NEO dominant selectable marker using the plasmid pJAF1 as templates [44]. The $5^{\prime}$ or $3^{\prime}$-region of the NEO-split marker containing gene disruption cassettes was amplified by primers TL235/TL20 or TL19/238, respectively, using the mixture of NEO marker and CMP1 5' region or $3^{\prime}$ region as templates, respectively. The overlap PCR fragments were purified, combined, and then biolistically transformed into the H99 or KN99a strains, as described previously [31]. Stable transformants were further confirmed on the YPD medium 
containing $200 \mathrm{mg} /$ liter G418. The cmp1 $\Delta$ mutants were further confirmed by diagnostic PCR (for primer sequences, see Table 2) and Southern blotting analysis.

To obtain the cmp1 $\triangle$ complemented strain, we amplified a $4.1 \mathrm{~Kb}$ genomic DNA fragment containing the $C M P 1$ 's upstream promoter region, open reading frame (ORF), and downstream terminator region using primers TL562/TL563 and cloned it into the plasmid pTBL1 to generate pTBL94 by infusion cloning. The resulting plasmid pTBL94, which contains the NAT selective marker, was digested by $S c a I$ and biolistically transformed into $c m p 1 \Delta$ mutants to generate the complemented strains.

To overexpress the Cryptococcus CMP1 gene, we linearized the plasmid pTBL92, which contains the GFP-CMP1 fusion construct under the histone $\mathrm{H} 3$ promoter's control, with $\mathrm{Xm} n \mathrm{I}$ and biolistically transformed it into the cmp $1 \Delta$ mutants. The overexpression of CMP1 was confirmed by qRT-PCR and fluorescence observation.

\subsection{Assays of Melanin and Capsule Production, and Mating}

Melanin formation was tested by dripping 50 microliters of each PBS-washed Cryptococcus overnight culture onto the Niger-seed agar plates. The Niger-seed plates were then placed at $30{ }^{\circ} \mathrm{C}$ or $37^{\circ} \mathrm{C}$ and incubated for two days, and the fungal colonies pigmentation was evaluated and photographed.

To investigate the capsule formation, we inoculated $10^{6}$ cells cultured in YPD medium overnight into MM medium and cultured at $30^{\circ} \mathrm{C}$ for $72 \mathrm{~h}$. The capsule's size was assessed, as previously described [39]. For matings, cell suspensions of cryptococcal mating partners ( $\alpha$ or a) were combined and cultured on V8 or MS medium in darkness at $25^{\circ} \mathrm{C}$. Formation of mating hyphae and chains of basidiospores were observed and recorded microscopically after incubation for two weeks.

\subsection{Virulence Studies}

Each strain's overnight culture was washed twice with PBS buffer and diluted to $2 \times 10^{6}$ cells $/ \mathrm{mL}$. Eight-week-old C57 BL/6 mice (female, $n=10$ /group) were intranasally inoculated with $10^{5} \mathrm{CFU}$ of each Cryptococcus strain, as described previously [45]. During the animal experiments, mice that were appeared to be in pain or moribund were euthanized by carbon dioxide inhalation. The log-rank test was used to calculate survival statistics between paired groups, and the nonparametric Mann-Whitney test with PRISM 7.0 (GraphPad Software, San Diego, CA, USA) was used for fungal load's statistical analyses.

\subsection{Fungal Loads and Histopathology of Infected Organs}

According to an animal protocol approved by Southwest University, the infected mice were euthanized at the endpoint of the animal experiment. For mice inoculated with the cmp1 $\Delta$ mutant, the animal experiment was stopped at $80 \mathrm{dpi}$. To compare the host inflammatory responses and fungal loads, we dissected the organs from the animals infected by each Cryptococcus strain at the animal experiments' endpoint. Infected organs were dissected and homogenized in PBS buffer with a tissue homogenizer. The homogenates were diluted, and 100 microliters of each diluent were dispersed on the YPD medium containing ampicillin and chloramphenicol. The CFUs of the infected organs were calculated after incubation at $30^{\circ} \mathrm{C}$ for three days. The infected organs were also sent to Servicebio (Servicebio, Wuhan, China) for section preparation after fixation in $10 \%$ formalin solution. Hematoxylin and eosin (H\&E)-stained tissue slides were observed under the light microscope.

\subsection{Cryptococcus-Macrophage Interaction and Serum Treatment Assay}

The Cryptococcus-macrophage interaction assay was carried out as described previously $[39,46]$. RAW246.7 murine macrophage cells were used in this study. After washing with PBS and opsonization with $20 \%$ mouse complement, a total of $2 \times 10^{5}$ cryptococcal cells of the overnight cultures of each Cryptococcus strain were added to each well containing RAW246.7 macrophage cells. After $2 \mathrm{~h}$ of coincubation at $37^{\circ} \mathrm{C}$, some of the cells were washed and fixed for phagocytosis assays as described previously [39]. Meanwhile, fresh DMEM was used to wash away the nonadherent extracellular 
cryptococcal cells, and the cultures were incubated for 0, 2, or 22 more hours. At various time points, distilled water was added to each well after removing the DMEM to lyse macrophage cells. The lysate was dispersed on YPD plates and yeast CFUs were counted to determine the phagocytosis rate and intracellular proliferation.

Serum treatment and Cryptococcus cell viability assay was performed as described previously [47]. At the indicated time points, aliquots were taken out and plated to YPD medium after serial dilution to determine cell viability.

Author Contributions: Conceptualization, T.-B.L.; data curation, L.-T.H. and L.W.; formal analysis, L.T.H. and T.-B.L.; funding acquisition, T.-B.L.; investigation, L.W.; methodology, L.W. and T.-B.L.; project administration, L.W.; resources, L.-T.H. and L.W.; supervision, T.-B.L.; validation, L.-T.H. and L.W.; writing-original draft, T.-B.L.; writing-review and editing, T.B.L.; All authors have read and agreed to the published version of the manuscript.

Funding: This study is supported by the National Natural Science Foundation of China $(31970145,31400133)$ and the Chongqing Research Program of Basic Research and Frontier Technology (cstc2017jcyjBX0034).

Acknowledgments: We acknowledge the use of the C. neoformans genome sequences at the Broad Institute.

Conflicts of Interest: The authors declare no conflict of interest.

\section{References}

1. Casadevall, A.; Perfect, J.R. Cryptococcus Neoformans; ASM Press: Washington, DC, USA, 1998.

2. Park, B.J.; A Wannemuehler, K.; Marston, B.J.; Govender, N.; Pappas, P.G.; Chiller, T.M. Estimation of the current global burden of cryptococcal meningitis among persons living with HIV/AIDS. AIDS 2009, 23, 525-530. [CrossRef] [PubMed]

3. Rajasingham, R.; Smith, R.M.; Park, B.J.; Jarvis, J.N.; Govender, N.P.; Chiller, T.M.; Denning, D.W.; Loyse, A.; Boulware, D.R. Global burden of disease of HIV-associated cryptococcal meningitis: An updated analysis. Lancet Infect. Dis. 2017, 17, 873-881. [CrossRef]

4. Kozel, T.R. Virulence factors of Cryptococcus neoformans. Trends Microbiol. 1995, 3, 295-299. [CrossRef]

5. Kronstad, J.W.; Jung, W.H.; Hu, G. Beyond the Big Three: Systematic Analysis of Virulence Factors in Cryptococcus neoformans. Cell Host Microbe 2008, 4, 308-310. [CrossRef] [PubMed]

6. Zaragoza, O. Basic principles of the virulence of Cryptococcus. Virulence 2019, 10, 490-501. [CrossRef]

7. Doering, T.L. How Sweet it is! Cell Wall Biogenesis and Polysaccharide Capsule Formation inCryptococcus neoformans. Annu. Rev. Microbiol. 2009, 63, 223-247. [CrossRef]

8. Taylor-Smith, L.M.; May, R.C. New weapons in the Cryptococcus infection toolkit. Curr. Opin. Microbiol. 2016, 34, 67-74. [CrossRef]

9. De Jesus, M.; Nicola, A.M.; Chow, S.-K.; Lee, I.R.; Nong, S.; Specht, C.A.; Levitz, S.M.; Casadevall, A. Glucuronoxylomannan, galactoxylomannan, and mannoprotein occupy spatially separate and discrete regions in the capsule ofCryptococcus neoformans. Virulence 2010, 1, 500-508. [CrossRef]

10. Biondo, C.; Messina, L.; Bombaci, M.; Mancuso, G.; Midiri, A.; Beninati, C.; Cusumano, V.; Gerace, E.; Papasergi, S.; Teti, G. Characterization of Two Novel Cryptococcal Mannoproteins Recognized by Immune Sera. Infect. Immun. 2005, 73, 7348-7355. [CrossRef]

11. Eigenheer, R.A.; Lee, Y.J.; Blumwald, E.; Phinney, B.S.; Gelli, A. Extracellular glycosylphosphatidylinositol-anchored mannoproteins and proteases ofCryptococcus neoformans. FEMS Yeast Res. 2007, 7, 499-510. [CrossRef]

12. Levitz, S.M.; Specht, C.A. The molecular basis for the immunogenicity ofCryptococcus neoformansmannoproteins. FEMS Yeast Res. 2006, 6, 513-524. [CrossRef] [PubMed]

13. E Vartivarian, S.; Reyes, G.H.; Jacobson, E.S.; James, P.G.; Cherniak, R.; Mumaw, V.R.; Tingler, M.J. Localization of mannoprotein in Cryptococcus neoformans. J. Bacteriol. 1989, 171, 6850-6852. [CrossRef] [PubMed]

14. Teixeira, P.A.C.; Penha, L.L.; Mendonça -Previato, L.; Previato, J.L. Mannoprotein MP84 mediates the adhesion of Cryptococcus neoformans to epithelial lung cells. Front. Cell. Infect. Microbiol. 2014, 4, 106. [CrossRef]

15. Mansour, M.K.; Yauch, L.E.; Rottman, J.B.; Levitz, S.M. Protective Efficacy of Antigenic Fractions in Mouse Models of Cryptococcosis. Infect. Immun. 2004, 72, 1746-1754. [CrossRef] 
16. Murphy, J.W. Influence of Cryptococcal Antigens on Cell-Mediated Immunity. Rev. Infect. Dis. 1988, 10 (Suppl. S2), S432-S435. [CrossRef]

17. Murphy, J. Protective cell-mediated immunity against Cryptococcus neoformans. Res. Immunol. 1998, 149, 373-386. [CrossRef]

18. Specht, C.A.; Nong, S.; Dan, J.M.; Lee, C.K.; Levitz, S.M. Contribution of Glycosylation to T Cell Responses Stimulated by RecombinantCryptococcus neoformansMannoprotein. J. Infect. Dis. 2007, 196, 796-800. [CrossRef]

19. Huang, C.; Nong, S.-H.; Mansour, M.K.; Specht, C.A.; Levitz, S.M. Purification and Characterization of a Second Immunoreactive Mannoprotein from Cryptococcus neoformans That Stimulates T-Cell Responses. Infect. Immun. 2002, 70, 5485-5493. [CrossRef]

20. Mansour, M.K.; Schlesinger, L.S.; Levitz, S.M. Optimal T Cell Responses toCryptococcus neoformansMannoprotein Are Dependent on Recognition of Conjugated Carbohydrates by Mannose Receptors. J. Immunol. 2002, 168, 2872-2879. [CrossRef]

21. Cadieux, B.; Lian, T.; Hu, G.; Wang, J.; Biondo, C.; Teti, G.; Liu, V.; Murphy, M.E.P.; Creagh, A.L.; Kronstad, J.W. The Mannoprotein Cig1 Supports Iron Acquisition from Heme and Virulence in the Pathogenic Fungus Cryptococcus neoformans. J. Infect. Dis. 2013, 207, 1339-1347. [CrossRef]

22. Reuwsaat, J.C.V.; Motta, H.; Garcia, A.W.A.; Vasconcelos, C.B.; Marques, B.M.; Oliveira, N.K.; Rodrigues, J.; Ferrareze, P.A.G.; Frases, S.; Lopes, W.; et al. A Predicted Mannoprotein Participates inCryptococcus gattiiCapsular Structure. mSphere 2018, 3, e00023-18. [CrossRef]

23. Viudes, A.; Lazzell, A.; Kirkpatrick, W.R.; Patterson, T.F.; Perea, S.; Pemán, J.; Martinez, J.P.; Loópez-Ribot, J.L. The C-terminal antibody binding domain ofCandida albicansmp58 represents a protective epitope during candidiasis. FEMS Microbiol. Lett. 2004, 232, 133-138. [CrossRef]

24. Inukai, T.; Nagi, M.; Morita, A.; Tanabe, K.; Aoyama, T.; Miyazaki, Y.; Bard, M.; Nakayama, H. The mannoprotein TIR3 (CAGL0C03872g) is required for sterol uptake in Candida glabrata. Biochim. Biophys. Acta (BBA)-Mol. Cell Biol. Lipids 2015, 1851, 141-151. [CrossRef]

25. Cao, L.; Chan, C.-M.; Lee, C.; Wong, S.S.-Y.; Yuen, K.-Y. MP1 Encodes an Abundant and Highly Antigenic Cell Wall Mannoprotein in the Pathogenic Fungus Penicillium marneffei. Infect. Immun. 1998, 66, $966-973$. [CrossRef]

26. Woo, P.C.Y.; Lau, S.K.P.; Lau, C.C.Y.; Tung, E.T.K.; Chong, K.T.K.; Yang, F.; Zhang, H.; Lo, R.K.C.; Cai, J.-P.; Au-Yeung, R.K.H.; et al. Mp1p Is a Virulence Factor in Talaromyces (Penicillium) marneffei. PLoS Negl. Trop. Dis. 2016, 10, e0004907. [CrossRef]

27. Basenko, E.Y.; Pulman, J.A.; Shanmugasundram, A.; Harb, O.S.; Crouch, K.; Starns, D.; Warrenfeltz, S.; Aurrecoechea, C.; Stoeckert, J.C.J.; Kissinger, J.C.; et al. FungiDB: An Integrated Bioinformatic Resource for Fungi and Oomycetes. J. Fungi (Basel) 2018, 4, 39. [CrossRef]

28. Perfect, J.R.; Ketabchi, N.; Cox, G.M.; Ingram, C.W.; Beiser, C.L. Karyotyping of Cryptococcus neoformans as an epidemiological tool. J. Clin. Microbiol. 1993, 31, 3305-3309. [CrossRef]

29. Nielsen, K.; Cox, G.M.; Wang, P.; Toffaletti, D.L.; Perfect, J.R.; Heitman, J. Sexual Cycle of Cryptococcus neoformans var. grubii and Virulence of Congenic a and $\alpha$ Isolates. Infect. Immun. 2003, 71, 4831-4841. [CrossRef]

30. Price, M.S.; Nichols, C.B.; Alspaugh, J.A. The Cryptococcus neoformans Rho-GDP Dissociation Inhibitor Mediates Intracellular Survival and Virulence. Infect. Immun. 2008, 76, 5729-5737. [CrossRef]

31. Fan, C.-L.; Han, L.-T.; Jiang, S.-T.; Chang, A.-N.; Zhou, Z.-Y.; Liu, T.-B. The Cys2His2 zinc finger protein Zfp1 regulates sexual reproduction and virulence in Cryptococcus neoformans. Fungal Genet. Biol. 2019, 124, 59-72. [CrossRef]

32. Lee, S.C.; Heitman, J. Function of Cryptococcus neoformans KAR7 (SEC66) in Karyogamy during Unisexual and Opposite-Sex Mating. Eukaryot. Cell 2012, 11, 783-794. [CrossRef] [PubMed]

33. Levitz, S.M.; Nong, S.-H.; Mansour, M.K.; Huang, C.; Specht, C.A. Molecular characterization of a mannoprotein with homology to chitin deacetylases that stimulates $\mathrm{T}$ cell responses to Cryptococcus neoformans. Proc. Natl. Acad. Sci. USA 2001, 98, 10422-10427. [CrossRef] [PubMed]

34. Kainz, K.; Bauer, M.A.; Madeo, F.; Carmona-Gutierrez, D. Fungal infections in humans: The silent crisis. Microb. Cell 2020, 7, 143-145. [CrossRef] [PubMed]

35. Kabir, V.; Maertens, J.A.; Kuypers, D. Fungal infections in solid organ transplantation: An update on diagnosis and treatment. Transplant. Rev. 2019, 33, 77-86. [CrossRef] [PubMed] 
36. Hasim, S.; Coleman, J.L. Targeting the fungal cell wall: Current therapies and implications for development of alternative antifungal agents. Futur. Med. Chem. 2019, 11, 869-883. [CrossRef]

37. A Wang, Z.; Li, L.X.; Doering, T.L. Unraveling synthesis of the cryptococcal cell wall and capsule. Glycobiol. 2018, 28, 719-730. [CrossRef]

38. Xue, C.; Tada, Y.; Dong, X.; Heitman, J. The Human Fungal Pathogen Cryptococcus Can Complete Its Sexual Cycle during a Pathogenic Association with Plants. Cell Host Microbe 2007, 1, 263-273. [CrossRef]

39. Liu, T.-B.; Wang, Y.; Stukes, S.; Chen, Q.; Casadevall, A.; Xue, C. The F-Box Protein Fbp1 Regulates Sexual Reproduction and Virulence in Cryptococcus neoformans. Eukaryot. Cell 2011, 10, 791-802. [CrossRef]

40. Vij, R.; Cordero, R.J.; Casadevall, A. The Buoyancy ofCryptococcus neoformansIs Affected by Capsule Size. mSphere 2018, 3, e00534-18. [CrossRef]

41. Blackstock, R.; Murphy, J.W. Secretion of the C3 component of complement by peritoneal cells cultured with encapsulated Cryptococcus neoformans. Infect. Immun. 1997, 65, 4114-4121. [CrossRef]

42. Davidson, R.C.; Cruz, M.; Sia, R.A.; Allen, B.; Alspaugh, J.; Heitman, J. Gene Disruption by Biolistic Transformation in Serotype D Strains of Cryptococcus neoformans. Fungal Genet. Biol. 2000, 29, 38-48. [CrossRef]

43. Kim, M.S.; Kim, S.-Y.; Yoon, J.K.; Lee, Y.-W.; Bahn, Y.-S. An efficient gene-disruption method in Cryptococcus neoformans by double-joint PCR with NAT-split markers. Biochem. Biophys. Res. Commun. 2009, 390, 983-988. [CrossRef]

44. Fraser, J.A.; Subaran, R.L.; Nichols, C.B.; Heitman, J. Recapitulation of the Sexual Cycle of the Primary Fungal Pathogen Cryptococcus neoformans var. gattii: Implications for an Outbreak on Vancouver Island, Canada. Eukaryot. Cell 2003, 2, 1036-1045. [CrossRef] [PubMed]

45. Cox, G.M.; Mukherjee, J.; Cole, G.T.; Casadevall, A.; Perfect, J.R. Urease as a Virulence Factor in Experimental Cryptococcosis. Infect. Immun. 2000, 68, 443-448. [CrossRef]

46. Liu, T.-B.; Xue, C. Fbp1-Mediated Ubiquitin-Proteasome Pathway Controls Cryptococcus neoformans Virulence by Regulating Fungal Intracellular Growth in Macrophages. Infect. Immun. 2013, 82, 557-568. [CrossRef]

47. Kingsbury, J.M.; McCusker, J.H. Fungal Homoserine Kinase (thr1 $\Delta$ ) Mutants Are Attenuated in Virulence and Die Rapidly upon Threonine Starvation and Serum Incubation. Eukaryot. Cell 2010, 9, 729-737. [CrossRef]

Publisher's Note: MDPI stays neutral with regard to jurisdictional claims in published maps and institutional affiliations.

(C) 2020 by the authors. Licensee MDPI, Basel, Switzerland. This article is an open access article distributed under the terms and conditions of the Creative Commons Attribution (CC BY) license (http://creativecommons.org/licenses/by/4.0/). 